\title{
An audit of the cervical screening programme in the National Drug Treatment Centre (NDTC)
}

\author{
Maeve Haran ${ }^{1}$ (D) John R. Kelly ${ }^{2} \cdot$ Liam Kennedy $^{3} \cdot$ Kieran Hennigan $^{4} \cdot$ Huma Farid $^{5} \cdot$ Cristina Herteu $^{6} \cdot$ Anna Kreisel $^{7}$. \\ Shamus Salehin ${ }^{6} \cdot$ Marie O$^{\prime}$ Sullivan $^{6} \cdot$ Shay Keating $^{6} \cdot$ Jo-Hanna Ivers $^{8} \cdot$ Mike Scully $^{6}$
}

Received: 30 October 2020 / Accepted: 3 December 2020 / Published online: 15 January 2021

(C) Royal Academy of Medicine in Ireland 2021

\begin{abstract}
Background Women diagnosed with substance use disorders (SUDs) have higher rates of major medical conditions compared to women without SUDs. Cervical cancer is the second leading cause of cancer death in women aged 20-39 years worldwide and women with SUDs have an increased risk of cervical cancer compared to women without SUD. The National Drug Treatment Centre (NDTC) cervical screening programme, derived from the national CervicalCheck programme, offers free cervical screening to patients attending for treatment of SUDs.

Aims This study aimed to audit adherence to the NDTC Cervical Screening guidelines before and after the implementation of an awareness-raising educational intervention.

Methods The electronic clinical records of women aged between 25 and 60 years attending the lead consultant's (M.S.) outpatient clinic were reviewed for documentary evidence indicating that information about the cervical screening programme had been discussed. This was completed before and one month after the implementation of an awareness-raising educational intervention.

Results All women ( $n=46$, mean age 36.3 ( $\mathrm{SD}=6.5$ ) years) had an opioid use disorder; $85 \%$ had a benzodiazepine use disorder, and $24 \%$ had an alcohol use disorder. Of these, $80 \%$ had at least one chronic medical condition, $76 \%$ had a psychiatric disorder, and 59\% were homeless. Adherence to the NDTC cervical screening guideline, as indicated by documentary evidence in clinical records, was $33 \%(14 / 43)$ at baseline, and rose to $88 \%(36 / 41)(p<0.0001)$ one month after the intervention.

Conclusions This completed audit cycle shows that an awareness-raising educational intervention can significantly improve adherence to a cervical screening programme in women with SUDs.
\end{abstract}

Keywords Cervical intraepithelial neoplasia $\cdot$ Cervical screening $\cdot$ Opioid-related disorders $\cdot$ Substance-related disorders $\cdot$ Uterine cervical neoplasms $\cdot$ Women's health

Maeve Haran

maeveharan@gmail.com

1 Daughters of Charity Disability Services, Navan Road, Dublin 7, Ireland

2 Trinity Centre for Health Sciences, Tallaght University Hospital, Dublin 24, Ireland

3 Psychiatry of Later Life, Sarto House, Sarto Road, Naas, Co. Kildare, Ireland

4 General Adult Psychiatry, University College Hospital Galway, Newcastle Rd, Galway, Ireland
5 General Adult Psychiatry, Belfast City Hospital, 51 Lisburn Road, Belfast BT9, 7AB 1841, Ireland

6 The HSE National Drug Treatment Centre, 30-31 Pearse Street, Dublin 2, Ireland

7 Adult Psychiatry Department, Aulingatan 22 C, SE-271 39 Ystad, Sweden

8 Department of Public Health and Primary Care, Institute of Population Health, School of Medicine, Trinity College Dublin, the University of Dublin, Russell Building, Tallaght Cross West, Tallaght, Dublin D24 DH74, Ireland 


\section{Introduction}

Substance use disorders (SUDs), particularly opioid use disorders, are associated with substantial disease burden [1]. Women with SUDs have higher rates of tobacco smoking [2]; sexually transmitted infections (STIs), including HIV and human papillomavirus (HPV) compared to women without SUDs [3, 4]; and specific and different healthcare needs compared to men [5]. HPV infection is one of the strongest risk factors for the development of both cervical pre-cancer and cancer [6, 7]. In addition, women with SUDs have an increased risk of cervical intraepithelial neoplasia and cervical cancer compared to women without SUD, which may be related to sexual risk behaviours and associated risk of HPV [8].

Cervical cancer, although the second leading cause of cancer death in women aged 20-39 years worldwide $[9,10]$, is one of the most preventable and treatable forms of cancer due to its prolonged pre-cancerous phase that can last 10-20 years [11]. In Ireland, between 2012 and 2015, there were 262 cases of invasive cervical cancer diagnosed per year with an age standardised incidence rate of 10.7 per 100,000 per year for this period [12].

CervicalCheck is a national cervical screening programme introduced in Ireland in 2008, which offers free cervical screening to women aged between 25 and 65 years. Despite major failings and an ongoing review process [13-15], CervicalCheck has provided tests to 1.8 million women from 2008 to 2018 [14]. During these 10 years, CervicalCheck has detected and treated over 100,000 cases of abnormal cervical cells. Moreover, the number of women who have been diagnosed with cervical cancer decreased by $7 \%$ year-on-year from 2010 to 2015 [16]. However, one in five Irish women do not take advantage of cervical screening [16] and major disparities exist in the burden of invasive cervical cancer incidence, with a consistent excess of $20 \%$ in the most socially deprived areas compared to the rest of the country [12].

The National Drug Treatment Centre (NDTC) is the longest and largest provider of specialist addiction treatment in Ireland. It provides an integrated, person-centred service and is part of the Health Service Executive. In 2008, 1108 people were assessed or entered treatment programmes in the NDTC; of these, $97 \%$ identified opioids as their primary problem substance [17]. Overall, in Ireland, 10,316 patients were prescribed opioid substitution treatment for opioid dependence in 2017, of whom $30 \%$ were female [18]. In our study sample, opioid use disorder was the primary diagnosis in $100 \%$ of the patients, all of whom were prescribed opioid substitution treatment.

Adherence to health screening programmes is a critical component of an integrated multidisciplinary care strategy to mitigate health inequalities for women with SUDs [5]. In line with best practice cervical screening guidelines, the NDTC incorporated the CervicalCheck programme, to develop cervical screening guidelines for use within the NDTC. This guideline states that all of the women aged between 25 and 60 years should be provided with information about the benefits and limitations of the cervical screening test, invited for regular cervical screening, be informed of results, and be referred onwards if necessary [19].

\section{Aims}

This study aimed to audit the adherence to the NDTC Cervical Screening guidelines before and after the implementation of an intervention designed to increase awareness of the guideline among the multidisciplinary team (MDT) healthcare workers and patients.

\section{Methods}

\section{Audited standards}

The standards were drawn from the HSE National Drug Treatment Centre Cervical Smear Guidelines (November 2016) which were derived from the national CervicalCheck programme.

These guidelines' objectives are as follows:

- To identify and invite eligible women for a cervical smear test.

- To provide information to women about the benefits and limitations of the cervical smear test.

- To provide access to screening onsite.

- To inform of result and refer if necessary.

\section{Inclusion/eligibility criteria}

Inclusion criteria were women attending the lead consultant's (M.S.) outpatient clinic who were aged 25-60 years, as this was the CervicalCheck's eligibility criteria for screening at the time of the audit [20]. For those included, their electronic clinical records were examined for evidence that they had been informed about the cervical screening programme and provided access to screening. We checked if the cervical screen had been completed at the NDTC; if they were due a repeat screen at the NDTC and if so, was there documentation offering this; or was the patient referred or followed up elsewhere.

Over 1 day in October 2019, a retrospective review of the electronic clinical records of the lead consultant's (M.S.) outpatients was completed. All women aged between 25 and 60 years were included. Over 1 day in November 2019, an identical data-gathering process was repeated for all women aged between 25 and 60 years attending the lead consultant's 
(M.S.) outpatient clinic. This data was separately gathered by two independent investigators (M.H., L.K.) who carried out an in-depth review of care plans, electronically searched all clinical notes for the terms 'cervical', 'smear', and 'screen and used a data collection sheet that was developed for this audit.

\section{Statistical analysis}

A two-tailed chi-square test with Yates' correction in GraphPad was used to determine the difference in proportions between baseline data and re-audit.

\section{Intervention}

\section{MDT discussion}

The MDT, including the nurse practitioner (M.O'S.) and medical officer (S.K.), examined the baseline data. A consensus was formed that doctors and nurses should identify eligibility criteria, document this in the care plan, and document which facility was responsible for cervical screening: the NDTC, a HIV clinic, a GP, or a colposcopy clinic.

\section{Awareness-raising activity}

An awareness-raising educational information poster targeting NDTC clinicians was developed to raise awareness of the free cervical screening programme available to patients attending the NDTC. This poster was displayed throughout clinical areas in the NDTC. An additional awareness-raising educational information poster specifically targeting patients was developed and displayed throughout NDTC patient waiting areas, to increase awareness among patients of the NDTC cervical screening programme.

\section{Personalised patient educational sessions}

Each patient whose electronic clinical record was not adherent to the NDTC cervical screening guidelines was scheduled for a review by a psychiatry registrar to inform the patient of the result of the audit, the reason her clinical record was not adherent to guidelines, and to rectify the deficit in adherence to the NDTC guidelines.

\section{MDT educational session}

In October 2019, an education presentation was delivered to all MDT members in the NDTC. This included the presentation of baseline results from the first phase of the study, an update of the national cervical screening programme (CervicalCheck) and the NDTC cervical screening guidelines.

\section{Results}

\section{Characteristics of the study sample}

Sociodemographic details of the study sample are presented in Table 1, and clinical details of the group are presented in Table 2.

\section{Baseline data}

Forty-three eligible patients were identified. Of the 43 patients included in the first phase of the audit, 14 patients' clinical records $(33 \%)$ were entirely adherent to the NDTC guidelines: their clinical records had evidence that they had been identified as eligible and invited for a cervical screening test; they had been provided information about the cervical screening test; they had been provided access to screening; and if a test

Table 1 Sociodemographic characteristics

\begin{tabular}{ll}
\hline Sociodemographic characteristics & $\begin{array}{l}\text { Study sample }(n=46) \\
\text { Mean (S.D.) } / n(\%)\end{array}$ \\
\hline Age & $36.3(6.52)$ \\
Age group (years) & \\
25-34 & $19(41 \%)$ \\
$35-44$ & $20(43 \%)$ \\
$45-60$ & $7(15 \%)$ \\
Race/ethnicity & \\
Irish White & $44(96 \%)$ \\
Irish Traveller & $1(2 \%)$ \\
Other White & $1(2 \%)$ \\
Primary language English & $45(98 \%)$ \\
Education level & \\
Primary school or below & $17(37 \%)$ \\
Secondary school: junior certificate & $14(30 \%)$ \\
Secondary school: leaving certificate & $8(17 \%)$ \\
Some university or higher & $1(2 \%)$ \\
Unknown & $6(13 \%)$ \\
Marital status: ever married & $4(9 \%)$ \\
Number of children & \\
0 & $10(22 \%)$ \\
1-3 & $29(63 \%)$ \\
$>3$ & $6(13 \%)$ \\
Unknown & $1(2 \%)$ \\
Hccommodation status & \\
Living in stable accommodation & $37(80 \%)$ \\
Hnemployment & $23(50 \%)$ \\
Has a medical card & \\
\hline &
\end{tabular}


Table 2 Clinical characteristics

\begin{tabular}{ll}
\hline Clinical characteristics & $\begin{array}{l}\text { Study sample }(n=46) \\
n(\%)\end{array}$ \\
\hline $\begin{array}{l}\text { Primary substance use disorder } \\
\text { Opioid use disorder }\end{array}$ & $46(100 \%)$ \\
Comorbid substance use disorder & \\
Alcohol use disorder & $11(24 \%)$ \\
Benzodiazepine use disorder & $39(85 \%)$ \\
Comorbid psychiatric diagnosis & $35(76 \%)$ \\
History of depressive episode & $22(48 \%)$ \\
History of psychosis & $8(17 \%)$ \\
Diagnosis of personality disorder & $13(28 \%)$ \\
Management of comorbid psychiatric diagnosis & \\
Managed by NDTC & $22(63 \%)^{\mathrm{a}}$ \\
Managed by GP & $2(6 \%)^{\mathrm{a}}$ \\
Managed by general adult psychiatrist & $6(17 \%)^{\mathrm{a}}$ \\
No active management & $5(14 \%)^{\mathrm{a}}$ \\
Chronic medical illnesses & $37(80 \%)$ \\
HIV positive & $2(4 \%)$ \\
Current/historical diagnosis of hepatitis C & $19(41 \%)$ \\
Medication prescriptions & \\
Methadone & $43(93 \%)$ \\
Buprenorphine & $3(7 \%)$ \\
Antidepressants & $24(52 \%)$ \\
Antipsychotics & $15(33 \%)$ \\
Benzodiazepines/Z-hypnotics & $27(59 \%)$ \\
Known history of sexual trauma & $20(43 \%)$ \\
\hline
\end{tabular}

${ }^{\text {a }}$ Percent by total number of comorbid psychiatric diagnosis (total number $=35)$

was taken, they had been informed of the result and referred onwards if appropriate.

Of the 14 patients' clinical records that were adherent to guidelines, 3 patients' clinical records had documentation of a cervical screening test in NDTC with a follow-up appointment and results of the test shared with each patient; 1 patient's clinical record had documentation of a cervical screening test in prison prior to admission, and a repeat was not due; 3 patients' clinical records had documentation that they had declined to have a cervical screening test taken in NDTC, and 7 patients' clinical records had documentation that they were being followed up in another clinic.

Of the total, 43 patients, 29 patients' clinical records (67\%) were not adherent to the NDTC guidelines. Of these, 27 patients' clinical records contained no evidence that they were identified as eligible, invited for a cervical screening test, or provided information about the cervical screening test. Two patients' clinical records were identified as eligible, provided information about the cervical screening, and invited for a cervical screening test. However, of these, one had no documentary evidence that the cervical screen had been conducted and the other contained no evidence that the cervical recall had been conducted.

\section{Re-audit data}

There was a significant improvement in adherence to guidelines from baseline data (14/43) compared to re-audit (36/41), $\chi^{2}(1, N=42)=24.34, p<0.0001$ (Fig. 1). Following the baseline audit, 5 patients, of the original 43 , were discharged from the service, and 3 new patients began attending. This led to a total of 41 patients included in the re-audit. Of the 41 patients included in the re-audit, 36 patients' clinical records $(88 \%)$ were entirely adherent to the NDTC guidelines. Five patients' clinical records $(12 \%)$ were not adherent to the NDTC guidelines. Although not adherent to guidelines, all women had pending appointments or had defaulted from arranged appointments.

\section{Discussion}

In this study, we show that a customised awareness-raising educational intervention for both healthcare staff and patients can significantly improve adherence to cervical screening guidelines in the National Drug Treatment Centre.

It is well established that women with SUDs experience a range of health outcome disparities [21].

There is a higher risk of cervical intraepithelial neoplasia and cervical cancer among women with SUDs compared to women without SUDs, which may be related to sexual risk behaviours and associated risk of HPV [8]. It is noteworthy that almost half (43\%) of the women in our sample had a history of sexual trauma which may have influenced cervical screening coverage.

\section{Adherence to the National Drug Treatment Centre Cervical Screening Guidelines}

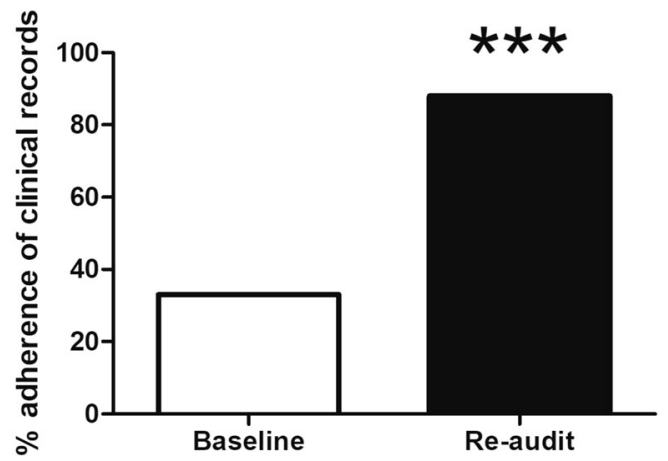

Fig. 1 The implementation of an awareness-raising education intervention in the National Drug Treatment Centre resulted in a significant improvement in adherence to Cervical Screening Guidelines one month after the intervention $(p<0.0001)$ 
Apart from increased disease burden [1], women with SUDs have higher rates of mental health comorbidities, with the prevalence of major depression being twice that of women in the general European population [22, 23]. A multicentre cohort study of people with opioid dependence disorder in Canada revealed that women with SUDs had a higher burden of physical and psychological health issues and had more childcare responsibilities compared to men with SUDs [5]. In our study, $80 \%$ of the women had a chronic medical illness despite most being younger than 45 years; $76 \%$ had a history of mental illness including $48 \%$ with a history of a depressive episode and $17 \%$ with a history of psychosis (see Table 2). Furthermore, $76 \%$ had given birth to at least one child (see Table 1).

Women with SUDs encounter increased levels of socioeconomic deprivation [24] and stigmatisation [25]. Indeed, stark levels of socioeconomic deprivation were evident in our sample, as indicated by a homelessness rate of 59\%, an unemployment rate of $96 \%$, together with $67 \%$ having poor formal education and not proceeding beyond the junior certificate (see Table 1).

While both men and women who use drugs must overcome stigmatisation when seeking treatment, stigmatisation appears to have more influence on women [26, 27]. Stigmatisation is associated with poor help-seeking behaviours and sexual health risks [28]. A qualitative study from the UK showed that women on opioid substitution therapy who experience stigma could feel disempowered and disregarded by healthcare systems, which may, in turn, lead to a negative impact on their sexual health behaviours and access to sexual health services such as screening clinics [28, 29]. Given the low rates of helpseeking and the various barriers to accessing treatment for this cohort, all opportunities for integration of broader physical, mental, and sexual health needs should be considered within the context of addiction treatment. Routine sexual health screening is an important part of harm reduction programmes and would significantly improve the health of women accessing specialist treatment. The current study offers a clear example of how this can be achieved.

An integrated healthcare approach for women with SUDs may mitigate some of their excess health burdens. In line with an integrated, person-centred, evidence-based approach, the NDTC adopted the CervicalCheck programme into its model of care, to develop cervical screening guidelines in 2016. Our baseline data identified deficiencies in the adherence to this guideline. Before our intervention, only one-third of patients eligible for cervical screening were identified and invited to participate in the NDTC cervical screening programme. This deficit emphasised the need for education of both healthcare staff and patients about the importance and benefits of participation in the cervical screening programme in the NDTC. Generally, clinician adherence to evidence-based guidelines can be poor [30] which may be due to lack of awareness of the guidelines, clinician's attitudes towards the guidelines, or a clinician lack the motivation to change behaviour. However, this can be improved by education, audit with feedback and by increasing integrated care pathways to aid shared decisionmaking between clinicians and patients to result in better quality healthcare [31, 32]. In our study, we designed the intervention to encourage greater awareness and perhaps demand among patients for cervical screening, and in parallel to this, we developed an educational intervention for the members of the MDT. By doing this, we sought to encourage a collaborative group dynamic to reinforce adherence to the guidelines as a collective in the delivery of care.

Our study highlighted the inefficiencies and fragmented nature of healthcare provision to women with SUDs. These deficiencies must be considered in the context of the wider cervical screening and healthcare system within which they occurred. For example, it was not fully or immediately clear where each woman had her routine cervical screen completed (the NDTC, STI/HIV clinics, colposcopy clinics, or GP practices) or what service was responsible for their ongoing monitoring. Coordinated and integrated care provision across addiction and mental and medical health services is a key component in advancing high-quality healthcare to women with SUDs [33-35]. The ability to record and share key information across care settings is vital for the provision of integrated, effective, and efficient personalised healthcare. The introduction of a national unified Electronic Healthcare Record (EHR) system would significantly advance this endeavour [36] across the whole healthcare system and there are some indicators that the response to the COVID-19 pandemic may expedite this process [37]. Moreover, information technology could be harnessed by clinicians and patients to facilitate more efficient and coordinated care pathways [31] that could improve outcomes for people with SUDs [38]. This would also facilitate regular audit of the adherence to national clinical guidelines.

Our audit also prompts consideration of the sensitive issues related to failures in the CervicalCheck screening programme. Gaps in the governance structures in the CervicalCheck programme and the non-disclosure of results were identified by the Scally Scoping Inquiry [13] and the Royal College of Obstetrics and Gynaecology [14]. The CervicalCheck Tribunal will consider a system for mandatory disclosure and the sustainable role of patient advocates in healthcare management and governance [15]. Also, an international review of the programme is planned.

An aligned public health programme that aims to reduce the incidence of cervical cancer is the HPV vaccination programme. The introduction of national HPV vaccination programmes has resulted in significant declines in HPV infection across Europe [39, 40]. In Ireland, the HPV vaccine was introduced into the national immunisation schedule for girls in the first year of second-level school in 2010 and was extended 
to boys in the first year of second-level school in 2019 [41]. However, similar to other countries, a cohort study in Ireland demonstrated that uptake of HPV vaccination is lower in disadvantaged schools [42] and policies must address this gap to ensure more equitable health outcomes among lower socioeconomic groups. If a high coverage of cervical screening and HPV vaccination can be achieved, research indicates that cervical cancer could be eliminated as a public health problem in Ireland and worldwide by 2069 , with the potential to prevent up to 13 million cases of cervical cancer globally [43].

While our study showed a significant improvement in the adherence to guidelines from 33 to $88 \%$ one month after the intervention, it is not yet known whether this will convert into a sustained improvement, or whether it can help increase awareness of other health issues in women with SUDs. Continuous monitoring, regular education of the MDTs, and further re-auditing are strategies that could be implemented at a local level in the NDTC to sustain and extend the improvements. Moreover, the existing NDTC cervical guidelines could be updated to reflect the most up to date eligibility criteria for cervical screening which have extended the screening age to 65 years and to necessitate that each woman has care plan documentation indicating eligibility and which facility is responsible for cervical screening: the NDTC, a HIV clinic, a GP, or a colposcopy clinic.

\section{Conclusions}

In summary, this audit that reviewed adherence to the NDTC guidelines on cervical screening demonstrated that targeted interventions to increase awareness about CervicalCheck among MDT healthcare workers and patients could lead to a significant increase in adherence to cervical screening guidelines.

More broadly, it highlighted the value of systematic audit of the adherence to clinical guidelines. Similarly, it indicated the need to examine other areas where improvements may be employed to mitigate the health burden for people with SUDs. These might include the development of an integrated, coordinated healthcare approach and the exploitation of digital health to optimise healthcare provision and to deliver greater equity for people with SUDs.

\section{Recommendations (see Table 3)}

We would recommend the adoption of a periodic audit of adherence to care guidelines to identify where improvements need to be made and to ensure a sustained increase in compliance to the guidelines. It would be beneficial to quantify the number of completed cervical screening tests among eligible patients to analyse if the increased adherence to guidelines had an impact on the uptake of cervical screening tests and
Table 3 Summary of recommendations

Recommendations

- Periodic audit of adherence to care guidelines.

- Check if guideline adherence impacted the uptake of cervical screening in this group.

- Introduce an education session into the NCHD induction programme.

- Revise and update the NDTC cervical screening guidelines.

- Introduce a national unified EHR system.

coverage in this group. We recommend the continuous education of clinicians by introducing an education session into the NCHD induction programme. We would also recommend revising and updating the NDTC cervical screening guidelines. Finally, this study highlights the fragmented nature of healthcare provision to women with SUDs and thus the importance of developing more coordinated and integrated care provision across addiction and mental and medical health. We recommend the introduction of a national unified EHR system which would significantly advance this endeavour.

\section{Limitations}

Information regarding the number of completed cervical screening tests following the implementation of targeted interventions was not gathered in this study. This prevents us from identifying if these interventions affected the uptake of cervical screening and prevent us from calculating the coverage of cervical screening in this group.

\section{Compliance with ethical standards}

Conflict of interest The authors declare that they have no conflicts of interest.

Ethical standards Approval of the study protocol was granted by The National Drug Treatment Centre Ethics Committee and conducted in accordance with the ICH Guidelines on Good Clinical Practice, and the Declaration of Helsinki.

\section{References}

1. Bahorik AL, Satre DD, Kline-Simon AH, Weisner CM, Campbell CI (2017) Alcohol, cannabis, and opioid use disorders, and disease burden in an integrated health care system. J Addict Med 11(1):3-9. https://doi.org/10.1097/adm.0000000000000260

2. Weinberger AH, Funk AP, Goodwin RD (2016) A review of epidemiologic research on smoking behavior among persons with alcohol and illicit substance use disorders. Prev Med 92:148-159. https://doi.org/10.1016/j.ypmed.2016.05.011

3. Phelan DF, Gange SJ, Ahdieh-Grant L, Mehta SH, Kirk GD, Shah K, Gravitt P (2009) Determinants of newly detected human papillomavirus infection in HIV-infected and HIV-uninfected injection 
drug using women. Sex Transm Dis 36(3):149-156. https://doi.org/ 10.1097/OLQ.0b013e31818d3df3

4. Ramsey SE, Bell KM, Engler PA (2010) Human immunodeficiency virus risk behavior among female substance abusers. J Addict Dis 29(2):192-199. https://doi.org/10.1080/10550881003684756

5. Bawor M, Dennis BB, Varenbut M, Daiter J, Marsh DC, Plater C, Worster A, Steiner M, Anglin R, Pare G, Desai D, Thabane L, Samaan Z (2015) Sex differences in substance use, health, and social functioning among opioid users receiving methadone treatment: a multicenter cohort study. Biol Sex Differ 6:21. https://doi. org/10.1186/s13293-015-0038-6

6. Schiffman M, Castle PE, Jeronimo J, Rodriguez AC, Wacholder S (2007) Human papillomavirus and cervical cancer. Lancet 370(9590):890-907. https://doi.org/10.1016/S0140-6736(07) 61416-0

7. Subramanya D, Grivas PD (2008) HPV and cervical cancer: updates on an established relationship. Postgrad Med 120(4):7-13. https://doi.org/10.3810/pgm.2008.11.1928

8. Kricker A, Burns L, Goumas C, Armstrong BK (2013) Cervical screening, high-grade squamous lesions, and cervical cancer in illicit drug users. Cancer Causes Control 24(7):1449-1457. https:// doi.org/10.1007/s10552-013-0224-y

9. Bray F, Ferlay J, Soerjomataram I, Siegel RL, Torre LA, Jemal A (2018) Global cancer statistics 2018: GLOBOCAN estimates of incidence and mortality worldwide for 36 cancers in 185 countries. CA Cancer J Clin 68(6):394-424. https://doi.org/10.3322/caac. 21492

10. Siegel RL, Miller KD, Jemal A (2020) Cancer statistics, 2020. CA Cancer J Clin 70(1):7-30. https://doi.org/10.3322/caac.21590

11. World Health Organisation (2014) In: Comprehensive cervical cancer control: a guide to essential practice, vol 2 nd edition. WHO Guidelines Approved by the Guidelines Review Committee. Geneva

12. National Cancer Registry Ireland (2017) Cancer trends 35 Cervical cancer. https://www.ncri.ie/sites/ncri/files/pubs/ CervicalCaTrendsReport MP.pdf. Accessed 19/08/20

13. Scally G (2018) Scoping inquiry into the CervicalCheck Screening Programme

14. Health Do (2019) Expert panel review of cervical screening (RCOG). https://www.gov.ie/en/pressrelease/146dcc-minister-forhealth-publishes-report-of-the-independent-expert-panel/

15. Tribunal C (2020). https://www.cervicalchecktribunal.ie/.

16. CervicalCheck (2017) CervicalCheck Programme Report 20162017

17. Drug Treatment Centre Board (2008) Drug Treatment Centre Board Annual Report 2008

18. European Monitoring Centre for Drugs and Drug Addictions (2019) Ireland Country Drug Report 2019. https://www.emcdda. europa.eu/publications/country-drug-reports/2019/ireland. Accessed 18/09/20

19. HSE National Drug Treatment Centre (2016) Cervical Smear Guidelines

20. Cervical Check (2020) Eligibility framework for cervical screening. Health Service Executive. https://www.cervicalcheck.ie/cervicalscreening-test-guidelines-and-forms/taking-a-cervicalscreeningtest.16276.html. Accessed 19/08/20 2020

21. Kulesza M, Matsuda M, Ramirez JJ, Werntz AJ, Teachman BA, Lindgren KP (2016) Towards greater understanding of addiction stigma: intersectionality with race/ethnicity and gender. Drug Alcohol Depend 169:85-91. https://doi.org/10.1016/j.drugalcdep. 2016.10.020

22. EMCDDA (2015) Comorbidity of substance use and mental disorders in Europe. Lisbon

23. Tuchman E (2010) Women and addiction: the importance of gender issues in substance abuse research. J Addict Dis 29(2):127-138. https://doi.org/10.1080/10550881003684582
24. The European Monitoring Centre for Drugs and Drug Addiction (2006) Annual report 2006. Selected Issue 2: a gender perspective on drug use and responding to drug problems. Luxembourg: European Monitoring Centre for Drugs \& Drug Addiction

25. United Nations Office on Drugs and Crime (2004) Substance abuse treatment and care for women: case studies and lessons learned. Vienna: United Nations Office on Drugs and Crime

26. Green CA (2006) Gender and use of substance abuse treatment services. Alcohol Res Health 29(1):55-62

27. Azim T, Bontell I, Strathdee SA (2015) Women, drugs and HIV. Int J Drug Policy 26:S16-S21

28. Medina-Perucha L, Scott J, Chapman S, Barnett J, Dack C, Family H (2019) A qualitative study on intersectional stigma and sexual health among women on opioid substitution treatment in England: implications for research, policy and practice. Soc Sci Med 222: 315-322. https://doi.org/10.1016/j.socscimed.2019.01.022

29. Women's Health Council (2009) Women \& substance misuse: drug misuse and women's health in Ireland

30. McGlynn EA, Asch SM, Adams J, Keesey J, Hicks J, DeCristofaro A, Kerr EA (2003) The quality of health care delivered to adults in the United States. N Engl J Med 348(26):2635-2645. https://doi. org/10.1056/NEJMsa02261531

31. Runnacles J, Roueche A, Lachman P (2018) The right care, every time: improving adherence to evidence based guidelines. Arch Dis Child Educ Pract Ed 103(1):27-33. https://doi.org/10.1136/ archdischild-2017-31274032

32. Beamish L, Sagorin Z, Stanley C, English K, Garelnabi R, Cousineau D, Barrios R, Klimas J (2019) Implementation of a regional quality improvement collaborative to improve care of people living with opioid use disorder in a Canadian setting. BMC Health Serv Res 19(1):663. https://doi.org/10.1186/s12913-019$4472-8$

33. Lawrence D, Kisely S (2010) Inequalities in healthcare provision for people with severe mental illness. J Psychopharmacol 24(4 Suppl):61-68. https://doi.org/10.1177/1359786810382058

34. Weisner C, Mertens J, Parthasarathy S, Moore C, Lu Y (2001) Integrating primary medical care with addiction treatment: a randomized controlled trial. JAMA 286(14):1715-1723. https://doi. org/10.1001/jama.286.14.1715

35. Naylor et al (2017) Mental health and new models of care. Lessons from the vanguards. The Kings Fund

36. Health Df (2013) e-Health Strategy for Ireland. https://www. ehealthireland.ie/StrategicProgrammes/Electronic-Health-RecordEHR-/

37. O'Carroll A, Duffin T, Collins J (2020) Saving lives in the time of COVID-19. Case study of harm reduction, homelessness and drug use in Dublin, Ireland. The London School of Economics and Political Science

38. Tofighi B, Abrantes A, Stein MD (2018) The role of technologybased interventions for substance use disorders in primary care: a review of the literature. Med Clin North Am 102(4):715-731. https://doi.org/10.1016/j.mcna.2018.02.011

39. Public Health England (2020) Surveillance of type-specific HPV in sexually active young females in England, to end 2018. HPR Volume 14 Number 2

40. Kjaer SK, Nygård M, Sundström K, Dillner J, Tryggvadottir L, Munk C, Berger S, Enerly E, Hortlund M, Ágústsson Á I, Bjelkenkrantz K, Fridrich K, Guðmundsdóttir I, Sørbye SW, Bautista O, Group T, Luxembourg A, Marshall JB, Radley D, Yang YS, Badshah C, Saah A (2020) Final analysis of a 14-year long-term follow-up study of the effectiveness and immunogenicity of the quadrivalent human papillomavirus vaccine in women from four nordic countries. (2589-5370 (Electronic))

41. Immunisation Guidelines, Chapter 10-human papillomavirus (2019) HSE National Immunisation Office. https://www.hse.ie/ eng/health/immunisation/hcpinfo/guidelines/ 
42. Migone C, Barrett T, Cotter S, Clarke A, Corcoran B (2017) The uptake of human papillomavirus vaccine in Irish schools: the impact of disadvantage. Irish medical journal (0332-3102 (Print))

43. Simms KT, Steinberg J, Caruana M, Smith MA, Lew JB, Soerjomataram I, Castle PE, Bray F, Canfell K (2019) Impact of scaled up human papillomavirus vaccination and cervical screening and the potential for global elimination of cervical cancer in 181 countries, 2020-99: a modelling study. Lancet Oncol 20(3): 394407-394407. https://doi.org/10.1016/S1470-2045(18)30836-2

Publisher's note Springer Nature remains neutral with regard to jurisdictional claims in published maps and institutional affiliations. 